\title{
Information System Framework Architecture for Organization Agnostic Logistics Utilizing Standardized IoT Technologies
}

\author{
Dimitris Karadimas \\ Industrial Systems \\ Institute/RC Athena, \\ Platani Patras, Greece \\ Email: \\ karadimas@ieee.org
}

\author{
Elias Polytarchos \\ Athens University of \\ Economics and Business, \\ Athens, Greece \\ Email: \\ e.polytarchos@gmail.com
}

\author{
Kyriakos Stefanidis \\ Library \& Information \\ Center, University of \\ Patras, Greece \\ Email: \\ stefanidis@ece.upatras.gr
}

\author{
John Gialelis \\ Industrial Systems \\ Institute/RC Athena, \\ Platani Patras, Greece \\ Email: \\ gialelis@isi.gr
}

\begin{abstract}
Logistics or supply-chain services provide enterprises and organizations with the necessary level of flexibility and efficiency in order to retain competitiveness under the increasingly turbulent e-business area. WebServices are utilized by organizations in order to integrate high and low level applications, thus providing a collaborative environment without affecting inter- and intra-enterprise processes. Nevertheless, the above context should be enhanced in order to comply with the Web-of-Things concept. This paper describes a sustainable approach towards the above requirement by employing ONS based services able to provide targeted information regarding RFID-enabled physical objects that are handled in an organization agnostic collaborative environment.
\end{abstract}

\section{INTRODUCTION}

$\mathrm{R}$ ADIO Frequency Identification (RFID) technology has already delivered revolutionary aspects in various areas such as logistics (supply chains), e-health management and materials identification / traceability. RFID technology itself allows an object's identification with effectiveness and efficiency. However traceability of an object calls for a robust and reliable system operating seamlessly over its entire lifecycle. Such a traceability system has to be implemented so as: a) its data model allows unique object identification and scalable, often big-data, databases, b) its underlying framework supports interoperability and c) its mechanism is capable to achieve end-to-end tracing providing full history information.

Despite RFID technology's nature in tracking, there are several challenges that need to be addressed. Since an RFID tag can be read from a quite long distance without requiring line-of-sight, it is possible that collisions may occur whereas also multiple tags could be read simultaneously. Therefore, there is no guarantee that a single tag will be consecutively detected on consecutive scans. Moreover, the use of RFID

This work was financially supported by the General Secretariat for Research and Technology (GSRT) [16] of the Hellenic Ministry of may constitute a serious threat for the information privacy, as it could be easily facilitated to espionage or unauthorized requests.

Based on the previously described situation there is a real need for an underlying framework able not only to support and complement the tracing functionality offered by the RFID technology but also to take into consideration the relevant tracking information of a physical object through its entire lifecycle in a secure and effectively protected way.

The work presented in this paper focuses in the challenging concept of an RFID-enabled, organization unaware, logistics management, by introducing an architecture that utilizes components of the Electronic Product Code (EPC) global network, such as the Object Naming Services (ONS) and the EPC Information Services (EPCIS), in order to support the Internet of Things concept. Our implementation is capable of enhancing the architecture of e-business frameworks, thus introducing an innovative collaborative business model which seamlessly integrates the inter-enterprise (public) with the intra-enterprise (private) processes.

The implemented architecture is demonstrated by the presentation of a case study in documents (books, papers, etc.) tracking and management in an academic library environment. Despite the fact that such an environment has quite lot variations from an e-business logistics environment, it has the potential to illustrate (and, even, simulate) the keypoints of the presented architecture when not a standalone but a whole network of academic libraries are taken into account.

\section{CURRENT STATUS}

Traceability is defined as the ability to trace the history, application or location of an entity, by means of recorded identifications. It also may be defined in general as the ability to trace and follow any product through all stages of production, processing and distribution. Traceability itself can be divided into three types:

- $\quad$ Back traceability (supplier traceability)

Development in the framework of project SELIDA - contract \#09SYN-72646 which runs under the "Cooperation", 2009 Call. 
- Internal traceability (process traceability)

- Forward traceability (end-user traceability)

Having the end-to-end traceability encompasses all three types of traceability and since traceability is defined over every stages of a value-chain, several researchers have pointed out various elements that should be taken into account.

Traceability systems store information and show the path of a particular object of interest along the whole value-chain from the supplier/producer to the retailer/distributor and eventually to the consumer/end-user. Throughout this process, secure, reliable and automatic object identification is crucial to provide effective and efficient tracing.

Barcode technology, in the past, has been used for the identification of items. However, in order to meet the traceability requirements imposed by the governments, a new technology that allows automated recording of information was needed. This need has been partially fulfilled by the revolutionary developments regarding the RFID technology.

Many logistic services have already integrated RFID identification technology into their services and products but these solutions most often implement a custom or proprietary communication flow. This means that it is quite difficult to come up with a generic approach against these solutions.

On the other hand, providing traceability services apart from trading logistics services i.e. physical documents interchange is even more demanding since existing services (e.g. Xerox Docushare, Papyrus, etc.) refer only to digitized documents management. Nowadays, document interchange between organizations, authorities and citizens is realized via the well-known courier shipping services (i.e. FedEx, etc.), but these services are almost always built into proprietary protocols while a gap often occurs when different services, even of the same type, in inter-continental transactions are involved.

Especially for book tracking (i.e. lending libraries, etc.) libraries and Inter-Librarian Loan (ILL) services in general employ standard interchange formats, exploited via web services, in order to share repositories and establish collaboration among them. However, a global standard elaborating libraries worldwide does not exist.

\section{ARCHITECTURE OF THE DEPLOYED INFORMATION SYSTEM}

In the context of this section we are going to concisely present the architecture of the Document Tracking System (DoTS), which has been designed in order to tackle the issues mentioned in the previous section.

Section A concisely describes the technologies employed whereas section B presents a brief description of the entire architecture and its basic components.

\section{A. Technologies and specifications employed}

The following technologies and specifications have been utilized in the context of the system: RFID, used to uniquely identify physical objects and EPC, providing the underlying framework that the system takes advantage of in order to offer standardized tracking services.

a) RFID

The RFID (Radio Frequency IDentification) is a welldocumented [1]-[6] and widely adopted [7], [8] technology, that provides the ability to uniquely identify objects tagged with RFID tags using special readers [9]. The main goal of the architecture is to be able to track documents on a potentially global scale. RFID provides significant advantages over other automatic ID technologies (specifically the widely applied bar and QR codes), as RFID tags:

- can be detected in bulk

- don't need to be aligned with the reader (line of sight) in order to be read

- can be detected from a greater distance

- have a larger data capacity

- $\quad$ are less susceptible to damage

These outweigh the benefits of the bar and QR code tags (which are less expensive and quite ubiquitous compared to RFID tags) for the application on important documents and can enable the introduction of innovative services, such as real time traceability and theft prevention. Additionally, RFID is intrinsic in the framework of global standards published by the GS1 that concern the EPC, which have been exploited in order to provide a method to globally provide tracking information services.

\section{b) EPCglobal}

The GS1 EPC global is a suite of standards and specifications that leverage the RFID technology in order to globally enable visibility and collaboration on an item level. These standards comprise the framework depicted in Fig. 1.

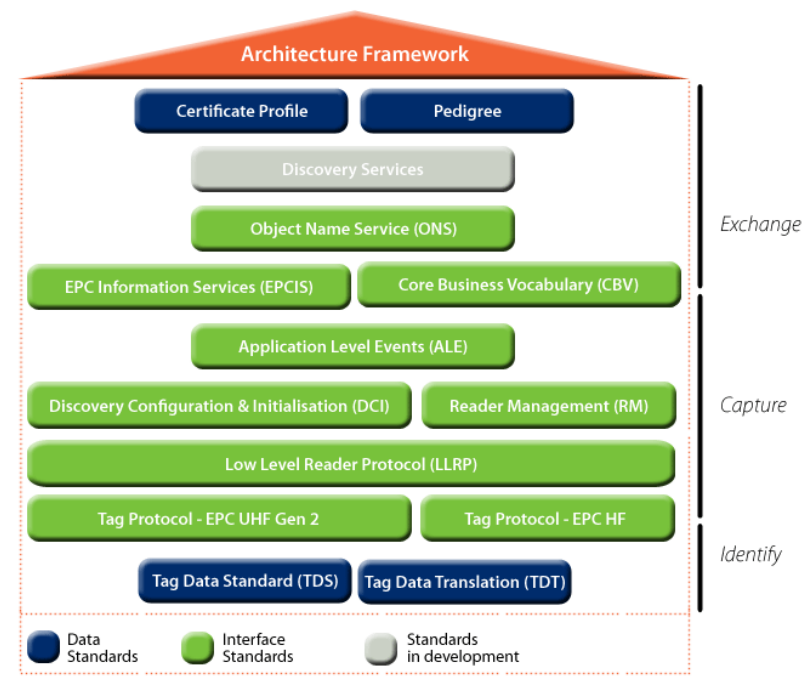

Fig. 1 GS1, EPCglobal framework standards, 2014 [6]

Information about these standards can be found in the GS1 website [5]. In the context of the proposed system, most of the EPC related GS1 standards have been utilized. 
c) Object Name Service Specification

The discovery and tracking service of physical documents that has been implemented exploits the Object Name Service (ONS) 2.0.1 [5] and the EPC Information Services (EPCIS) 1.0.1 [2], in order to enable the mapping of EPC tagged documents to addresses of arbitrary, but with a standardized interface, object management services (OMS).

\section{B. Framework Architecture}

The proposed framework aims to support as many of the EPC global standards as possible; in order to provide the ability to map single physical objects to URIs and to track related information regarding the entire lifecycle of the representation for all involved organizations in the value chain, together with the realization of ONS-based web services available in the cloud.

The proposed architecture is value chain agnostic pertaining:

- common logistics value-chain (i.e. manufacturer, logistics service, retail and end-user/customer)

- physical documents inter-change value-chain between public authorities or organizations of the public sector and citizens or companies of the private sector

- objects inter-change value-chain in demanding cases such as insurance organizations, shipping companies, courier companies, etc.

Fig. 2 illustrates the incorporation of the aforementioned technologies in the proposed framework architecture.

The proposed ONS service layer consists of a collection of ONS-based web services that are able to provide information from the organization's internal hierarchy model breakdown, using the global EPC notation, without affecting the existing processes of the value chain. Each organization's ONS service layer is responsible for providing per object, both public and private information, using the global EPC notation.

The private web services satisfy per sector- needs for realtime, synchronous physical objects tracking. These needs have various orientations, depending on the corresponding node of the chain. The public web services address needs of common operations, regarding single object's lifecycle information, such as location, history etc.

The integration of the RFID subsystem, into the architecture framework raises two main issues. The first regards the capturing of tags' information as well as the identification of the tags themselves. The RFID reader scaling, range and reading angle are of major importance since it is incorporated in a versatile environment (many different types of organizations performing totally different internal processes). The second issue regards security and privacy issues that are raised when the identified object's data should be classified. Security and privacy issues are presented in detail in next paragraph.

\section{a) RFID Middleware}

The RFID middleware is responsible for receiving, analyzing processing and propagating the data collected by

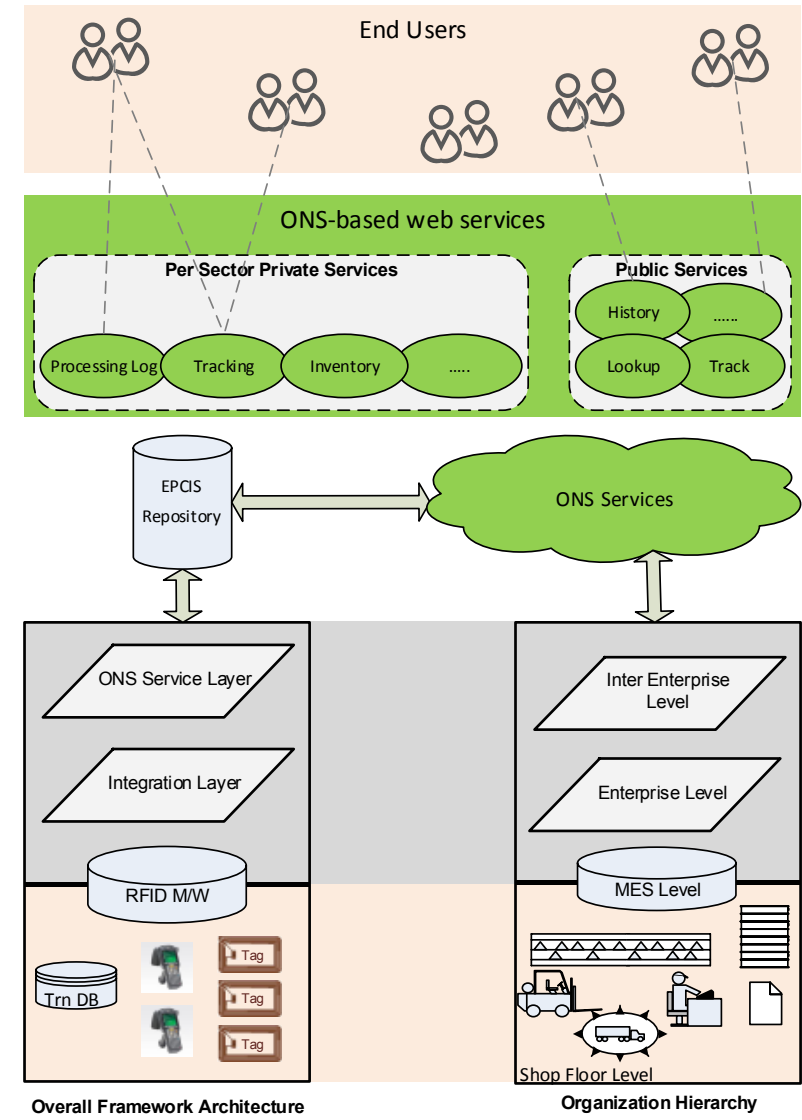

Fig. 2. Proposed framework architecture.

the RFID readers to the Information System that supports the business processes. The middleware hides the complexity of the actual RFID infrastructure and only provides business events. On the other hand, the middleware is oblivious of how the data it provides gets handled afterwards.

Specifically, the RFID middleware provides facilities for:

1. EPC Allocation

2. Device Management and Monitoring

3. Data Collection and Integration

4. Data Structure and Data Association

5. Data Filtering and Data Routing

6. Line Coordination and Process Control

7. Legend and Graphics Creation

8. Visibility and Reporting

9. Track and Trace Applications

\section{b) ONS Resolver}

The purpose of the ONS Resolver is to provide secure access to the ONS infrastructure, so that its clients would not only be able to query for the OMSs related to EPCs (which is the de facto use case of the ONS), but also introduce new or delete any existing OMSs for the objects. This has been accomplished by creating a SOAP web service layer that functions on top of the ONS, which provides authenticated and authorized users with the capability to query the whole 
ONS infrastructure and discover the OMSs for the given EPCs, to add or delete OMSs or to add or delete users of the ONS Resolver, depending on their permissions.

In addition to the secure access to the ONS infrastructure, the ONS Resolver acts as an authorization server [10] for the relevant OMSs. This way, whenever a user uses the ONS Resolver to get the address of an OMS and authenticates successfully, an access token will be returned along with the result of the query, which, if used in the subsequent interaction between the user and the OMS, it can provide privileged access to the service. The authorization procedure that has been developed is depicted in Fig. 3.

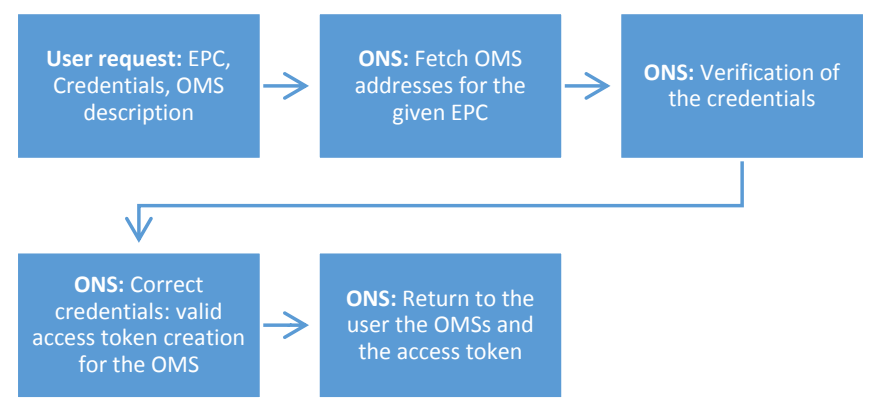

Fig. 3. ONS authorization procedure.

c) Object Management Services

The object management services (OMS) provide management, tracking and other value added services for the EPC tagged objects. The ONS Resolver maps the object management services to the objects according to their owner and type (for example the OMS for objects tagged using an SGTIN EPC is going to be determined by their GTIN; i.e. the OMS for the EPCs with the same GTIN is common [5]) and they should be implemented according to the EPCIS specification [2].

The most common case of the aforementioned context is the presentation of all historical data relevant to a single physical object. The resulting historical data can contain information regarding object transactions within locations controlled by the owner of the item or other organizations, as long as all the implicated parties implement the described framework; this was rendered possible through the utilization of the ONS Service Layer. The data of the objects stored by the different parties adhere to the same API and, as a result, one can select the exact kind of these data that are to be presented. For example historical data could describe an object's transaction only with dates on interaction with an RFID M/W, or with geographic interpreted location of the interaction, or even with more meta-data of the object like its purpose of transportation, original owner, final destination and whether it is classified or not. In an expanded version of multiple organizations running the presented framework, any tagged object (document, package, suitcase, etc.) could be easily and reliable recognized in the entire framework's context.
Finally, the arbitrary nature of the OMS themselves, even though they are implemented with standardized formation, enables each organization to level-up objects' related information according to the specific organization needs and requirements.

d) Security and Privacy aspects

Since the proposed architecture is based on web services, our first goal is to identify and classify those services in regards to their security requirements. Even before that, we can safely assume that all web services can and should implement TLS as a standard form of encrypting the data channel in use (usually the Internet).

Based on the aforementioned architecture diagram, we can easily identify that there are public and non-public facing web services. In regards to the non-public facing web services, the ones that reside within the internals of the proposed architecture stack, we can exploit the useful fact that both the clients and the servers of this part of the architecture are known and can be controlled in regards to their implementation of the security mechanisms. Therefore it is safe to assume that the use of client certificates is a feasible security mechanism. Client certificates are a very robust way of handling secure and, in conjunction with TLS, encrypted authentication and authorization and the issues with scalability and deployment that are usually encountered in more general scenarios are not applicable to our proposed architecture. As for the public facing web services, we follow the industry standard of API keys due to the fact that although we name those services "public facing", in reality those services will be accessed not by casual end users but by the information systems of the organizations that will employ that services of our proposed architecture. A case study of such a deployment is described in the next chapter.

Moreover, all the web services should follow a standard of secure design that, although already a common practice in popular web services around the web, we will briefly describe below.

As mentioned above, all services should be authenticated over an encrypted communication channel. Messages should be digitally signed, as well as encrypted, to provide privacy and tamper-proofing when the message travels through intermediary nodes route to the final destination even within different organizations that implement the same proposed architectural stack. The usage of the access token (a unique ID or nonce, a cryptographically unique value) generated by the ONS Resolver within every request, will, obviously, provide protection against unauthorized usage and it will also aid to the detection message replay and man in the middle attacks. HTTP methods should be valid for each API key and associated resource collection and method by white-listing allowable methods. Any request for exposed resources should be protected against CSRF and insecure direct object references should be avoided. 


\section{CASE Study On PhysicAl DocUments TraCKIng IN A LIBRARY ENVIRONMENT}

In order to evaluate the applicability of the proposed architecture on a real environment we deployed it on the existing Integrated Library System (ILS) that is being used in the central university library of Patras, Greece. The ILS that is being used by the institution is the well-known open source ILS named KOHA [11].

As with all ILS, KOHA supports a variety of workflows and services to accommodate the needs of the institution. Our proposed scheme focuses on a handful of those services and augments them with additional features. This is generally done by adding, in a transparent way, the additional UI elements and background processes that are needed for our scheme to work.

The specifics on how this is done will be presented in the following section, while first we will discuss briefly on the exact services of the KOHA ILS that our scheme aims to augment.

\section{A. Supported services}

In its initial design, our scheme aims to provide additional functionality on the core services of an ILS. Those services are:

- Check Out

- Check In

- New Record

- Delete Record

There are also a number of tracking services that our scheme aims for and those are:

- History

- Location

- Search

To elaborate, when the check-out or check-in services are called in KOHA, an additional call is made on the SELIDA (the name of the developed framework from the related project) middleware that updates the status of the affected documents on the SELIDA database. The details on how those calls are made will be described in the next section. Similar functionality can be seen on the entire core and tracking services that are applicable in our scheme as described in the previous chapters.

\section{B. Integration layer}

In order to provide the added functionality to the existing KOHA services we designed and implemented an integration layer that seamlessly handles all the extra work along with the usual service workflow.

The primary reason to provide a seamless layer instead of changing the actual services (i.e. the source code) is the fact that every integrated system that is actually in a production environment needs the benefits of a continuous and stable update process that is offered by the systems development team along and implemented by the organization's administrator. Adding extra functionality in the form of changes to the system's code would require continuous maintenance of this part of the system on par with the normal updates of the KOHA ILS.

To overcome this obstacle, and given to the fact that $\mathrm{KOHA}$ is a web based application as most of the modern ILS, we designed the integration layer so that it is injected upon page load as a JavaScript file on the pages that we are interested in. On our specific case study, we used the "mod_substitute" directive on the Apache web server that was serving the KOHA web pages. Each time a module/page of interest is requested by the server (i.e. check-out), the web server adds a <script $>$ tag that loads all the additional functionality in the form of a JavaScript file.

This layer, in the form of a JavaScript application module, adds the required UI elements for our proposed scheme to work and handles all the web service requests that are necessary.

As an example, we will showcase the check-out process. When the user navigates to the check-out KOHA module by requesting the module's URL, the apache server injects the selida.js file which is the integration layer code (so called SELIDA module). SELIDA module starts executing upon page load and adds the button "Scan" next to the button "Checkout". When the user presses the button "Scan", a web service request is launched from the SELIDA module which starts up the RFID reader via the SELIDA middleware services. The results (per example the barcodes and titles of the documents that the reader identified) are communicated back to the SELIDA module which in turn shows a pop-up window to the user indicating those results. After this procedure, the normal check-out workflow resumes by sending the required POST requests to the KOHA web server (just like when the user presses the "Checkout" button after adding the barcode). When the check-out process ends and the web server responds with the next web page as per KOHA workflow, the SELIDA module sends a second web service request to the middleware indicating that the check-out is complete so that the rest of SELIDA architecture continues with its own check-out workflow presented in the previous chapters.

As we can see on this example, the extra steps that are needed for our scheme to work are completely transparent from the ILS itself. KOHA as an application retains its original functionality and workflow while the user benefits from the added services that our SELIDA module provides.

Fig. 5 illustrates the framework's activities during the first object identification phase, previously described.

\section{Object Management Services}

Since RESTful [12] web services are becoming the most common developers' choice for implementing API's and data retrieval services SELIDA's services have been designed following the RESTful architectural style and JavaScript Object Notation (JSON) [13] for data exchanging. Although these technologies do not comply with a specific standard, as other technologies do, i.e. SOAP, XML web services, when combined together they constitute a lightweight datainterchange mechanism that is easy for humans to read and 


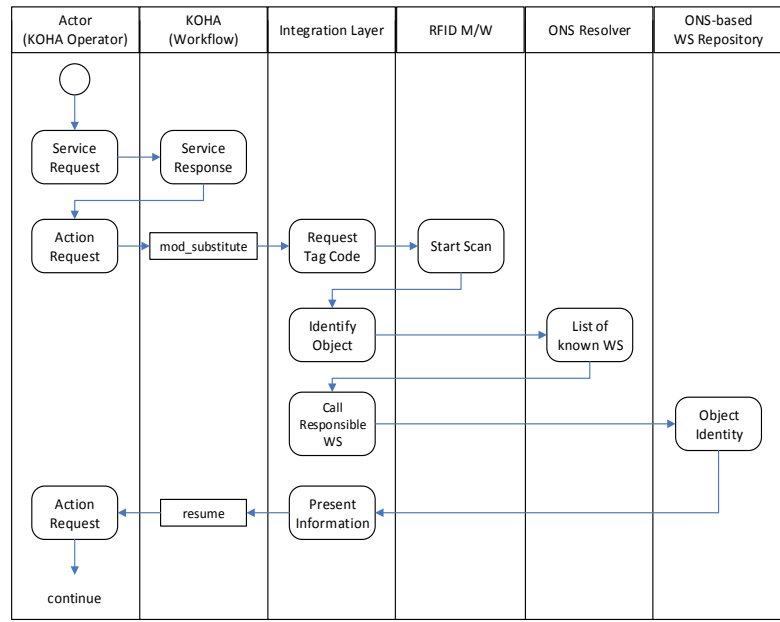

Fig. 5. Framework's activities during an object identification phase.

write and also easy for machines to parse and generate, thus appearing to be the easiest and most comprehensive messaging way among web services. Moreover, nowadays numerous open standards-like specifications (i.e. JSONDoc [14], Swagger [15], etc.) have been presented towards describing, producing, consuming, and visualizing RESTful web services so as RESTful web services can become a complete framework.

Currently, based on the deployment of the proposed architecture on the existing Integrated Library System (ILS) that is being used in the central university library of Patras, Greece, three types of management services (history, location

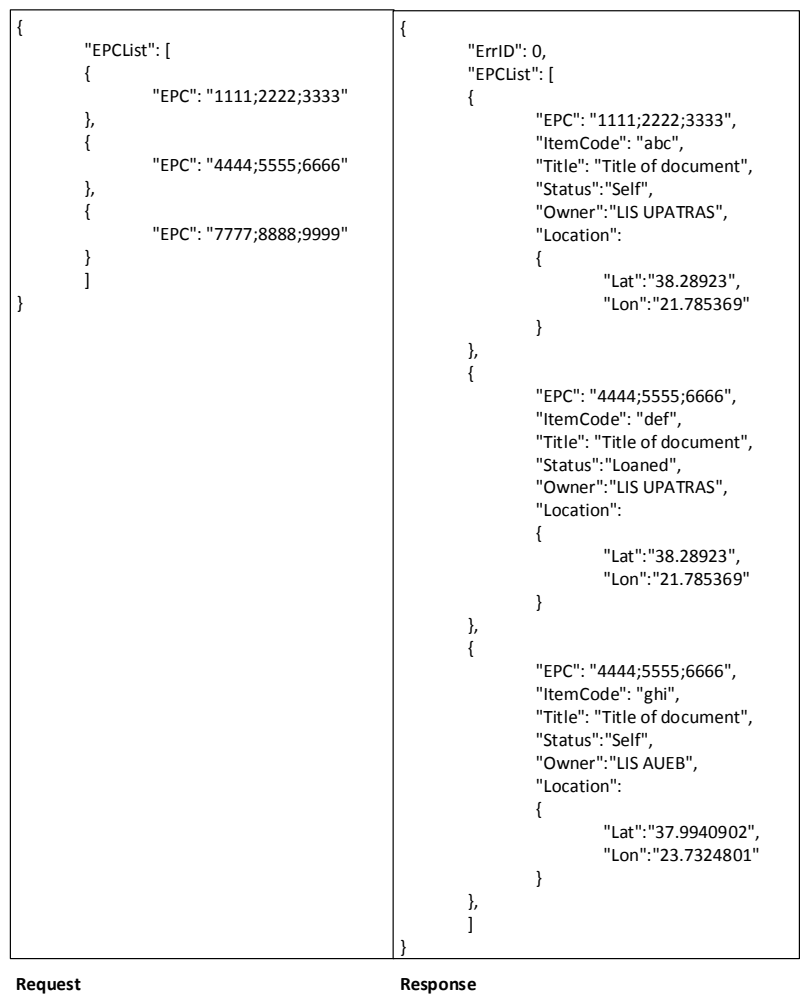

Fig. 4. An example of JSON request and response data structure for status OMS. and search) have been implemented, as described in paragraph A.

As for an example, the JSON schemes for the request and response of the search service is presented in Fig. 4.

As depicted in Fig. 4 request to the status OMS may contain multiple EPC tags at a time, while the implemented system response contains an indicative error code ( 0 in case of no error) and relevant data for recognized books. In the illustrated example the first two EPCs belong to books owned by the central university library of Patras, Greece (LIS UPATRAS) while the third belongs to a book owned by the university library of Athens University of Economics and Business, Athens, Greece (LIS AUEB).

\section{V.BENEFITS AND SCALABILITY}

Although the described framework has been deployed in an ILL (Inter-Librarian Loan) environment its initial inspiration and design has been originated in general logistics or warehouse inventory stocktaking environments. Based on this origin the whole framework has been designed and deployed so as to offer organization agnostic information, supported by ONS-based web-services that are integrated at the top of each organization's hierarchy stack, as illustrated in Fig. 2.

The proposed architecture offers a set of versatile characteristics due to the combination of reliability and uniqueness, induced by RFID technology along with the ONS perspective implementation throughout the architecture that derives interoperable information exchange. These characteristics could constitute the basis for the employment of such a framework so as to derive into a generic InternetOf-Things service platform able to handle information and processes of any value-chain type, including and not limited at food supply chain, luggage handling, physical document interchange, etc.

This prospect could be further substantiated by the nature of the ONS, which is actually a mechanism that leverages Domain Name System (DNS) to discover information about an object and its related services from the EPC code. Conclusively, the presented architecture could be scaled in the same way as internet does, since internet is based on DNS and the presented architecture is based on ONS, inheriting DNS scalability capabilities.

\section{CONCLUSION}

In spite of the promising nature of RFID technology, numerous applications in the actual logistics field area have not been reported. Only a few pilot studies as well as experimental tests have proved that RFID would be a successful tool to enable supply chain traceability. The reasons why companies are yet reluctant to have confidence in adopting the technology to gain their product visibility may be attributed to the several challenges such as lack of standards, immaturity of RFID, and privacy issues.

This paper presents a ubiquitous approach towards a collaborative logistics environment and concludes with a case study implementation involving physical documents tracking 
in an academic library. The main focus of the proposed methodology and implemented architecture addresses the issue of empowering the whole framework with a standard specification for objects tracking services, thus the integration of the ONS-perspective in the architecture. The integration itself, which, within the EPC global framework is mainly achieved by utilizing the Object Naming Service, enables involved organizations to act agnostically of their entities and provides them with the ability to resolve EPC tagged objects to arbitrary services, but with a standardized manner.

However, security and privacy issues should be further investigated as future work, apart from the issues already covered though the implementations of the framework's web services, so as the presented framework will be promising enough for evolutionizing the way currently exploited for tracking items in the supply chain.

\section{REFERENCES}

[1] ISO. (2010). ISO RFID Standards: A Complete List. Retrieved from http://rfid.net/basics/186-iso-rfid-standards-a-complete-list

[2] GS1. (2007). EPC Information Services (EPCIS) Version 1.0.1 Specification. Retrieved from http://www.gs1.org/gsmp/kc/ epcglobal/epcis/epcis_1_0_1-standard-20070921.pdf
[3] GS1. (2010). EPCglobal: Specifications. Retrieved from http://www.gs1.org/epcglobal/standards/specs

[4] GS1. (2010). EPCglobal: Standards. Retrieved from http://www.gs1.org/gsmp/kc/epcglobal

[5] GS1. (2013). Object Name Service (ONS). Retrieved from http://www.gs1.org/gsmp/kc/epcglobal/ons/ons_2_0_1-standard20130131.pdf

[6] GS1. (2014). EPCglobal framework standards. Retrieved from http://www.gs1.org/gsmp/kc/epcglobal

[7] Hossain, M. (2012). A Comparison of Voluntary and Mandatory Adoption of Radio Frequency Identification (RFID) Technology in Organizations. Retrieved from http://www.wbiconpro.com/421Hossain.pdf

[8] RFIDjournal. (2013). Survey Shows Half of U.S. Retailers Have Already Adopted Item-Level RFID. Retrieved from http://www.rfidjournal.com/articles/view?9168

[9] Wikipedia. (2007). Radio-frequency identification. Retrieved from http://en.wikipedia.org/wiki/Radio-frequency_identification

[10] IETF. (2012). RFC 6749: The OAuth 2.0 Authorization Framework. Retrieved from http://tools.ietf.org/html/rfc6749

[11] Official Website of Koha Library Software. Retrieved from http://koha-community.org/

[12] Wikipedia (2014). Representational state transfer. Retrieved from http://en.wikipedia.org/wiki/Representational_state_transfer

[13] JSON (JavaScript Object Notation). Retrieved from http://json.org

[14] JSONDoc. Retrieved from http://jsondoc.org

[15] Swagger. Retrieved from https://helloreverb.com/developers/swagger

[16] General Secretariat for Research and Technology, http://www.gsrt.gr 